\title{
¿Qué creencias sostienen estudiantes de pedagogía, profesorado en servicio y personal académico formador de docentes, en Chile, sobre la pobreza?'
}

\author{
What Beliefs Do Chilean Student Teachers, In-Service Teachers, and Teacher Educators Hold About Poverty? ${ }^{2}$
}

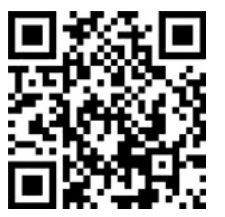

Viviana de Lourdes Gómez-Nocetti ${ }^{3}$
Pontificia Universidad Católica de Chile
Villarrica, Chile
vgomezn@uc.cl
http://orcid.org/0000-0002-4465-823X

Recibido 12 de noviembre de 2015 • Corregido 6 de noviembre de 2016 • Aceptado 28 de noviembre de 2016

\begin{abstract}
Resumen: El fracaso sistemático de la niñez de nivel socioeconómico bajo en la escuela es una preocupación compartida. ¿Qué papel juegan las creencias del profesorado y otras personas actoras en la mantención de este fracaso? Esta investigación responde a la pregunta: ¿Hay diferencias en las creencias de estudiantes de pedagogía, profesorado en servicio y personal académico formador de docentes en Chile acerca de la enseñanza y aprendizaje de alfabetización y matemática inicial en contextos de pobreza? Se aplicó un cuestionario con dos preguntas abiertas y dos escalas Likert a 265 estudiantes de pedagogía, 66 docentes en servicio y 25 docentes formadores de profesorado. Con los datos cualitativos se conformaron descripciones organizadas como "visiones" $y$ con los datos cuantitativos se analizaron conglomerados y ANOVA con base en los puntajes Z. Los resultados muestran 1) visión negativa de la escuela, la niñez y las familias vulnerables, 2) visión del buen profesor o profesora centrada en aspectos afectivo-motivacionales, 3) visión negativa del contexto familiar transversal a toda la muestra, 4) sobre el 70\% poseía una visión intermedia en creencias sobre alfabetización y un $50 \%$ en creencias sobre matemática inicial, 6) la visión menos presente fue la visión compleja, 7) el profesorado en servicio poseía las visiones más estereotipadas respecto a las escuelas vulnerables, 8 ) solo entre el $10 \%$ y el $30 \%$ de personal docente formador de profesorado poseía creencias complejas. Estos resultados informan a las instituciones formadoras de docentes acerca de las creencias y los actores a los que deben apuntar sus estrategias de cambio.
\end{abstract}

Palabras claves: Creencias; profesorado; pobreza; alfabetización; matemática; Chile.

\footnotetext{
${ }^{1}$ Este artículo corresponde a resultados parciales del proyecto Fondecyt n 1120550, financiado por el Fondo Nacional de Ciencia y Tecnología, Fondecyt de Conicyt.

${ }^{2}$ This article corresponds to partial results of the project Fondecyt $n^{\circ} 1120550$, financed by Conicyt- National Fund of Science and Technology, Fondecyt (by its acronym in Spanish).

${ }^{3}$ Doctora en Psicología Evolutiva y del Aprendizaje Escolar de la Pontificia Universidad Católica de Chile. Se desempeña actualmente como profesora asociada en el campus Villarrica. Anteriormente se desempeñó durante 20 años en la Facultad de Educación, donde realizó docencia en pre-grado, magister y doctorado en el área de educación. Además, ha sido jefa del Departamento de Psicopedagogía y subdirectora de Investigación en dicha Facultad. Actualmente dicta asignaturas de aprendizaje y desarrollo en la carrera de Pedagogía en Educación Parvularia. También desarrolla numerosos proyectos de investigación con fondos concursables a nivel nacional. Ha sido editora de sección de la revista Infancia y Aprendizaje y revisora de las revistas Teaching and Teacher Education; The Teacher Educator y Estudios Pedagógicos. Actualmente es la directora del Grupo de Estudio en Educación del Programa Fondecyt de Conicyt. Posee publicaciones en revistas de corriente principal nacionales e internacionales; también ha escrito 3 capítulos de libro en coautoría.
} 
doi: http://dx.doi.org/10.15359/ree.21-1.17

URL: http://www.una.ac.cr/educare

CORREO: educare@una.cr

\begin{abstract}
The systematic failure of low socioeconomic level children at school is a shared concern. What is the role of teachers and other involved individuals' beliefs in maintaining this failure? This research answers the question: Are there differences in the beliefs between student teachers, in-service teachers, and Chilean teacher educators about the teaching and learning of literacy, and early math in the context of poverty? A questionnaire with two open questions and two Likert scales were applied to 265 student teachers, 66 in-service teachers and 25 teacher educators. With qualitative data, some descriptions were organized as "visions"; and with quantitative data, conglomerates and ANOVA were analyzed, based on Z scores. The results show: 1) negative view on school, children, and vulnerable families, 2) good teacher vision centered in affective-motivational features, 3) negative view on the family context shared to the whole sample, 4) over $70 \%$ had an intermediate vision on literacy beliefs and $50 \%$ on initial mathematics beliefs, 6) complex vision was the less present, 7) in-service teachers had the most stereotypical views regarding vulnerable schools, 8) only between $10 \%$ and $30 \%$ of teacher educators had complex beliefs. These results inform teacher education institutions about the beliefs and actors to whom their strategies for change should target.
\end{abstract}

Keywords: Beliefs; teachers; poverty; literacy; mathematics; Chile.

\title{
Introducción
}

El fracaso escolar de la niñez proveniente de nivel socioeconómico bajo es una realidad en Chile y en el mundo. En nuestro país, la pobreza se concentra principalmente en seis de las quince regiones (Ministerio de Desarrollo Social, 2012), las que también exhiben bajos resultados en el sistema de medición nacional de la calidad (SIMCE). Cuando se comparan los resultados con consideración del nivel socioeconómico (NSE), estudiantes de los sectores NSE bajo obtienen, en promedio en matemáticas, 62 puntos menos que los de NSE alto en $4^{\circ}$ básico, ascendiendo a 72 puntos en $6^{\circ}$ básico. En lenguaje la distancia alcanza a 53 puntos en $2^{\circ}$ y $4^{\circ}$ básico y a 48 puntos en $6^{\circ}$ básico (Agencia de Calidad de la Educación, 2015). Dado que en los informes de resultados de la prueba SIMCE se especifica que una diferencia igual o mayor a 5 puntos es significativa, estos resultados llaman a la preocupación. ¿Cómo podemos explicar este fenómeno? ¿Cuáles podrían ser las acciones que debiéramos realizar desde la formación inicial del profesorado para mejorar estos resultados?

La bibliografía internacional señala que el profesorado que se desempeña en contextos de pobreza no se siente preparado y los programas de su formación no han abordado explícitamente la pobreza como uno de los contextos de intervención pedagógica (Cho, Convertino y KhoureyBowers, 2015; Hughes, 2010; Levin, 1995). Sin embargo, para solucionar este problema no solo se deben poseer estrategias o conocimiento específico, sino también unas creencias positivas y acordes con el cambio, pues las percepciones erróneas podrían frustrar los esfuerzos de equidad en las escuelas, por lo que examinar los estereotipos y actitudes del profesorado hacia sus estudiantes que viven en la pobreza resulta una tarea fundamental (Gorski, 2012). 
doi: http://dx.doi.org/10.15359/ree.21-1.17

URL: http://www.una.ac.cr/educare

CORREO: educare@una.cr

Nos preguntamos si, en Chile, las personas actoras responsables de ofrecer una educación sensible a las necesidades de la niñez proveniente de sectores de pobreza poseen unas creencias que permitan revertir los resultados anteriores o, de modo contrario, encontrar pistas para clarificar qué es lo que debemos modificar. En este artículo se exploran las creencias de tres importantes actores educativos: estudiantes de pedagogía, profesorado en servicio y también formadores y formadoras de docentes, sobre la alfabetización y la matemática inicial de los niños y las niñas que viven en situación de pobreza.

\section{Marco teórico}

Numerosas investigaciones abordan los obstáculos que enfrenta el cambio en los procesos de enseñanza y de aprendizaje en las escuelas vulnerables chilenas (Belleï, 2001; Román, 2003). Dentro de estas, los estudios sobre el pensamiento del profesorado destacan el poder de las creencias sobre su conducta (Román, 2003), expectativas y estrategias de enseñanza (Carlson, 2000; Jadue, 1997; Leyva, 2005; Raczynski y Muñoz, 2005; Román, 2006) y el grado de responsabilidad que este siente frente a los bajos logros de sus estudiantes (Carlson, 2000; Eyzaguirre, 2004).

Las creencias se entienden como "individual's judgement of the truth or falsity of a proposition [juicios individuales de la verdad o falsedad de una proposición]" (Pajares, 1992, p. 316). Estas son comprensiones, premisas o proposiciones de base subjetiva, acerca del mundo que se considera verdadero (Kagan, 1992; Richardson, 2003).

Pajares (1992) señala que las creencias se organizan en sistemas, poseen una función adaptativa y ayudan a definir y entender tanto al mundo como al autoconocimiento. Por su naturaleza afectiva, valorativa y episódica, las creencias se convierten en un filtro a través del cual se interpretan los nuevos fenómenos. Es cierto que los procesos de pensamiento pueden ser precursores y creadores de creencias, pero el efecto de filtro de la estructura de creencias, en última instancia, redefine, distorsiona o remodela el posterior procesamiento del pensamiento y de la información.

Por su naturaleza y origen, algunas creencias son más indiscutibles que otras. Incluso, se priorizan en función de sus conexiones o relación con otras creencias u otras estructuras cognitivas y afectivas, moderadas por la situación (Rodrigo, Rodríguez y Marrero, 1993). Las creencias se forman tempranamente y, mientras más pronto se incorporan a la estructura, más difícil es alterarlas. Estas tienden a autoperpetuarse, perseverando frente a las contradicciones (Pajares, 1992), por lo que son resistentes a pesar de la exposición al conocimiento científico. Por tanto, debido a su influencia en la organización del conocimiento e información y su papel crítico en el comportamiento de las personas, las creencias se han vuelto centrales en el estudio del pensamiento del profesorado. 
doi: http://dx.doi.org/10.15359/ree.21-1.17

URL: http://www.una.ac.cr/educare

CORREO: educare@una.cr

Lortie (1975) indica que los profesores y profesoras fueron aprendices por observación durante gran parte de su vida, al ser protagonistas directos en su propia escolarización, por lo que al ingresar a los programas de formación ya poseían creencias bastante consolidadas sobre la enseñanza, el papel del profesorado, el aprendizaje, la evaluación, etc., las que funcionan como marco interpretativo con que enfrentan su tarea educativa.

Las creencias del profesorado han sido descritasy caracterizadas en décadas anteriores (Joram \& Gabriele, 1998; Kagan, 1992; Nespor, 1987). w ejemplo, estos grupos confían poderosamente en los componentes afectivos de la enseñanza y el aprendizaje, minimizando los aspectos cognitivos; conciben la enseñanza como transmisión de cuerpos intactos de conocimiento desde el sujeto conocedor al aprendiz; tienden a explicar las situaciones basándose en el recuerdo de incidentes específicos y creen que algunas instancias están más allá del control de cada docente y que no están sujetas a cambio y, además, poseen una visión epistemológica objetivista del conocimiento (Elkind, 2004). Por otra parte, Wideen, Mayer-Smith y Moon (1998) señalan que muchas de estas creencias no son modificadas por el paso por los programas de formación inicial docente.

Un tipo particular de creencia es el estereotipo, el que refiere a las creencias sobre las personas y los comportamientos de grupo (Leyens, Yzerbyt y Schadron, 1994), en las que basamos nuestro comportamiento al enfrentar a las demás personas. El problema que presentan es que estas llegan a consolidarse como "naturales", denotando una "existencia subyacente" en los sujetos y en los grupos (Rothbart y Taylor, 1993, citados en Schadron e Yzerbyt, 1996). Según Armstrong (2010), los prejuicios parecen servir a las clases acomodadas para absolverse a sí mismas de la responsabilidad de mejorar las escuelas para estudiantes de más bajos ingresos. Al caricaturizar a los padres y madres como flojos, adictos o que no desean trabajar, no cuidan a los niños y niñas y no valoran la educación, se hace prevalecer una actitud clasista. Pero el clasismo está tan enraizado en las personas, que incluso estas no son conscientes de que lo son. Estas creencias llevan a expectativas más bajas en el profesorado y a una acción pedagógica que es consistente con dichas expectativas (Del Río y Balladares, 2010). Por ejemplo, los profesores y profesoras consideran mejores estudiantes, desarrollan expectativas más altas e, incluso, distribuyen en los cursos de rendimiento a aquellos niños y niñas bien vestidos y con apariencia de clase media (Jones y Vagle, 2013). Según Templeton (2011-2012), muchos profesores y profesoras tienden a ver el mundo a través de una "óptica de clase media", por lo que en contextos de pobreza tienden a connotar negativamente a sus estudiantes y a las familias de origen, con lo que afectan la calidad tanto de la enseñanza como del aprendizaje.

En Chile, existen pocos estudios que abordan las creencias relativas a educación y pobreza. En estudios sobre "los sectores pobres" $y$ "la pobreza", los sujetos caracterizan a ambos indistintamente en términos de "carencia" (Carlson, 2000; Denegri et al., 2010). Una investigación sobre expectativas ante el género y nivel socioeconómico realizada por Del Río y Balladares (2010) muestra que el estudiantado de pedagogía chileno tiende a atribuir características 
doi: http://dx.doi.org/10.15359/ree.21-1.17

URL: http://www.una.ac.cr/educare

CORREO: educare@una.cr

personales negativas a los niños y niñas de nivel socioeconómico bajo, tales como baja autoestima, inestabilidad y lentitud. Esto también es referido en estudios con profesorado en servicio en Chile (Cuadra, 2009; Román, 2003) y otros países latinoamericanos (Rosales, 2008).

La bibliografía sobre alfabetización inicial muestra creencias negativas en el profesorado en servicio respecto a estudiantes vulnerables. Este considera que el alumnado proveniente de sectores de pobreza posee limitadas funciones lingüísticas, escaso lenguaje y no tiene cultura (Condemarín, 2002; Stevens y Palincsar, 1992). Además, muestran una percepción de que el deterioro existente en los hogares provoca problemas emocionales que interfieren en la atención y concentración de los niños y niñas en la escuela. Por otra parte, debido al analfabetismo de las familias y por la falta de libros en casa, no podrían dar apoyo suficiente para desarrollar hábitos lectores en su progenie (Rosales, 2008). El personal docente privilegiaría enseñar valores antes que enseñar a leer, pues piensa que hablándoles cariñosamente se transforman en madres y enfermeras, para compensar lo que no tienen en casa (Stevens y Palincsar, 1992).

Respecto a la metodología, creen que la lectura debe enseñarse en forma lineal, yendo de lo simple a lo complejo (fonemas, decodificación y vocabulario); y la escritura consistiría en deletrear, poner mayúsculas, signos de puntuación y usar frases completas; además, esto ocurriría naturalmente después de aprender a leer (Añorve, 2011; Shaw y Mahlios, 2011; Stevens y Palincsar, 1992).

Respecto a la matemática, no existen investigaciones relativas a las creencias sobre el aprendizaje de la niñez en sectores de pobreza. Sin embargo, las creencias sobre la matemática han sido recogidas por numerosos estudios tanto con el profesorado como con estudiantes de pedagogía. En general, el profesorado considera la matemática como un conocimiento estático y estable (Zakaria y Musiran, 2010), que se entrega mediante presentaciones organizadas y clases expositivas (Swars, Hart, Smith, Smith y Tolar, 2007). La matemática es concebida como una disciplina con reglas y fórmulas que deben ser aprendidas por memorización. Además, es restringida, formal y rígida (Szydlik, Szydlik y Benson, 2003), lo que la hace aburrida, difícil y desagradable (Toluk-Uçar, Piskin, Akkaş, y Taşçi, 2010). Philipp et al. (2007) señalan que las creencias afectan la forma en que el profesorado piensa y enseña la matemática.

Respecto al estudiantado de pedagogía, Markovits (2011) señala que posee creencias más negativas hacia la naturaleza de las matemáticas que hacia la enseñanza de la disciplina. En cambio, el profesorado en servicio muestra creencias convencionales del aprendizaje, enseñanza (Gil y Rico, 2003) y naturaleza de la matemática, lo que influye en su forma de enseñar (Beswick, 2012; Kuntze, 2012), al determinar sus propósitos y afectar directamente el trabajo profesional, es decir, la planificación, la evaluación y la toma de decisiones durante las interacciones con sus estudiantes (Bryan y Atwater, 2002).

Romper el círculo vicioso que se produce entre las creencias negativas respecto a los niños y las niñas de niveles socioeconómicos bajos y sobre las posibilidades de alfabetización y aprendizaje 
doi: http://dx.doi.org/10.15359/ree.21-1.17

URL: http://www.una.ac.cr/educare

CORREO: educare@una.cr

de la matemática inicial que posee el estudiantado de pedagogía y el profesorado en servicio es un imperativo ético que deben asumir sus instituciones formadoras. En este sentido, la figura del formador y formadora de docentes es determinante puesto que en sus manos se encuentran tanto los espacios de la formación inicial como los de formación continua. En general, no se encuentran estudios respecto a las creencias de los grupos formadores de profesores y profesoras, por esta razón estos han sido incluidos como parte de la muestra de esta investigación.

\section{Pregunta y objetivos del estudio}

La pregunta que guió este estudio fue: ¿Hay diferencias en las creencias que sostiene el estudiantado de pedagogía, el profesorado en servicio y su personal formador acerca de la alfabetización y matemática inicial en contextos de pobreza? Los objetivos del estudio eran 1) identificar las creencias acerca de la enseñanza y aprendizaje sobre la niñez en contextos de pobreza que sostienen los grupos mencionados y 2) comparar las creencias de personas actoras responsables de llevar a cabo procesos educativos con calidad y equidad en nuestro país.

Con esto se buscaba levantar información para llevar al debate la tendencia a formar docentes generalistas para ambientes homogéneos, en los que la pobreza y las creencias sobre su impacto e importancia son ignoradas por los actuales programas de formación docente. Este estudio se realizó el año 2012, momento en que comenzaron a resurgir investigaciones sobre pobreza y educación en el mundo entero.

\section{Metodología}

Se realizó un estudio mixto para explorar las creencias, por lo que mediante la aplicación de instrumentos que contenían preguntas abiertas y escalas Likert, se realizaron tanto análisis cualitativos como cuantitativos de la información recopilada.

\section{Muestra}

Se aplicó un cuestionario a una muestra representativa de 265 estudiantes de pedagogía, 66 docentes en servicio y 25 formadores y formadoras de profesorado. El estudiantado de pedagogía cursaba programas de pedagogía ${ }^{4}$ en diversas universidades públicas y privadas a lo largo de Chile. La mayoría respondió al cuestionario en formato on-line mediante la plataforma

\footnotetext{
${ }^{4}$ Las carreras de pedagogía que cursaba el estudiantado involucrado en esta investigación fueron: Pedagogía General Básica (107 estudiantes); Pedagogía en Educación Parvularia (48 estudiantes); Educación Diferencial (69 estudiantes); Pedagogía en Enseñanza Media en Lenguaje (18 estudiantes) y Pedagogía en Enseñanza Media en Historia (23 estudiantes). La cantidad de estudiantes fue representativa del porcentaje de estudiantes por cada carrera en el nivel nacional.
} 
doi: http://dx.doi.org/10.15359/ree.21-1.17

URL: http://www.una.ac.cr/educare

CORREO: educare@una.cr

surveymonkey. Solo un pequeño grupo respondió en el formato de papel y lápiz. El profesorado seleccionado se desempeñaba en enseñanza básica en las tres regiones en las que se desarrolló este estudio: 27 en la región Metropolitana, 20 en la región de Los Lagos y 19 en la región de la Araucanía. Todos respondieron en formato de papel y lápiz. Los formadores y formadoras de profesorado pertenecían a las mismas instituciones que el estudiantado de pedagogía encuestado en este estudio. Los formadores y formadoras de docentes respondieron al cuestionario en un formato on-line.

El estudiantado del grupo mayoritario tenía entre 18-22 años (58.1\%), mientras que el $35.3 \%$ tenía entre $23-27$ años, el $4.5 \%$ tenía entre $28-32$ y el $1.9 \%$ entre $33-37$ años.

Entre el profesorado en servicio, el grupo más numeroso fue el que tenía entre 23-29 años (47.8\%), mientras que el $21 \%$ tenía entre 30-39 años, el 13\% entre $40-49$ años, el 13\% entre 5059 y el $4.3 \%$ entre 60 y 66 años.

Entre los formadores y formadoras de profesorado el grupo mayoritario fue el de 40-49 años (32.1\%) y el $28.5 \%$ entre $30-39$ años, mientras que el $14.2 \%$ fluctuaba entre $50-59$ años y el mismo porcentaje presentaba entre 60-69 años. Solo el 7\% tenía entre $26-29$ años. En relación con la formación académica, el $17.8 \%$ de los formadores y formadoras de profesores tenía solo título profesional, mientras que el $64.2 \%$ tenía grado de magíster y el restante $17.8 \%$ tenía grado de doctor.

\section{Instrumentos}

Se aplicaron 2 cuestionarios y 2 preguntas abiertas.

Cuestionario de creencias sobre alfabetización inicial: escala Likert en la que los grupos participantes debían responder marcando su opción de 1 a 6, en la que 1 significaba "no es cierto para mí" y 6 "muy cierto para mi". Todas las afirmaciones estaban redactadas desde una visión simple, por lo que una puntuación mayor indicaba creencias más ingenuas.

La escala estaba compuesta de 22 ítems que conformaron las 5 subescalas que se muestran en la Tabla 1.

Cuestionario de creencias sobre matemática inicial: escala Likert en la que los grupos participantes debían responder marcando su opción de 1 a 6, en la que 1 significaba "no es cierto para mí" y 6 "muy cierto para mi". Todas las afirmaciones estaban redactadas desde una visión simple.

La escala estaba compuesta por 24 ítems, los que conformaron las 6 subescalas que muestra la Tabla 2. 
doi: http://dx.doi.org/10.15359/ree.21-1.17

URL: http://www.una.ac.cr/educare

CORREO: educare@una.cr

Tabla 1: Descripción de las subescalas del Cuestionario de creencias sobre alfabetización inicial

\begin{tabular}{|c|c|c|}
\hline Factor & $\begin{array}{l}\text { Alfa de } \\
\text { Cronbach }\end{array}$ & Ejemplo de ítem \\
\hline Visión tradicional de la alfabetización & ,800 & $\begin{array}{l}\text { La lectura es un acto de decodificación alfabética que permite } \\
\text { comprender fielmente lo que está escrito en un texto. }\end{array}$ \\
\hline Visión actual de la alfabetización & ,713 & $\begin{array}{l}\text { Después de la lectura, en una clase de una escuela en } \\
\text { contexto de pobreza, es importante que los niños y niñas } \\
\text { logren ir más allá de las ideas del texto, aunque se pierdan } \\
\text { las ideas del autor o autora. }\end{array}$ \\
\hline $\begin{array}{l}\text { Prejuicio sobre alfabetización en } \\
\text { contextos de pobreza }\end{array}$ & 673 & $\begin{array}{l}\text { Es difícil que los niños y niñas provenientes de contextos } \\
\text { de pobreza aprendan a leer y escribir adecuadamente, } \\
\text { pues hablan mal. }\end{array}$ \\
\hline $\begin{array}{l}\text { Condiciones para la alfabetización en } \\
\text { contextos de pobreza }\end{array}$ & ,541 & $\begin{array}{l}\text { Si no hay libros en las casas de las familias en contextos de } \\
\text { pobreza, difícilmente se desarrollarán hábitos lectores en } \\
\text { los niños y niñas. }\end{array}$ \\
\hline $\begin{array}{l}\text { Explicaciones del éxito o fracaso en la } \\
\text { alfabetización en contextos de pobreza }\end{array}$ & ,514 & $\begin{array}{l}\text { Para que los niños y niñas que se educan en contextos } \\
\text { de pobreza aprendan a leer adecuadamente, lo más } \\
\text { importante es ensayar velocidad lectora (fluidez). }\end{array}$ \\
\hline
\end{tabular}

Tabla 2: Descripción de las subescalas del Cuestionario de creencias sobre aprendizaje y enseñanza de la matemática inicial

\begin{tabular}{|c|c|c|}
\hline Factor & $\begin{array}{c}\text { Alfa de } \\
\text { Cronbach }\end{array}$ & Ejemplo de ítem \\
\hline $\begin{array}{l}\text { Prejuicio sobre matemática y } \\
\text { pobreza }\end{array}$ & 800 & $\begin{array}{l}\text { En contextos de pobreza siempre habrá estudiantes que no } \\
\text { entenderán las matemáticas, no importa el método que se use. }\end{array}$ \\
\hline Representación sobre los pobres & 814 & $\begin{array}{l}\text { Los problemas emocionales de los niños y niñas de } \\
\text { sectores de pobreza no les permiten aprender matemática } \\
\text { adecuadamente en la escuela. }\end{array}$ \\
\hline $\begin{array}{l}\text { Formación del profesor o } \\
\text { profesora }\end{array}$ & 767 & $\begin{array}{l}\text { Enseñar matemática en contextos de pobreza requiere } \\
\text { conocimientos específicos de la disciplina por parte del } \\
\text { personal docente. }\end{array}$ \\
\hline Factores de éxito &, 535 & $\begin{array}{l}\text { Dar énfasis en la memorización de procedimientos, reglas } \\
\text { y símbolos es un factor clave en la enseñanza de las } \\
\text { matemáticas en contextos de pobreza. }\end{array}$ \\
\hline Determinismo de la pobreza & ,731 & $\begin{array}{l}\text { Los niños y niñas de sectores de pobreza necesitan más } \\
\text { ayuda para aprender matemática que los niños y niñas de } \\
\text { otros contextos. }\end{array}$ \\
\hline Aprendizaje de las matemáticas &, 635 & $\begin{array}{l}\text { Más que enseñar cómo resolver un problema matemático, es } \\
\text { mejor dar la oportunidad para que los niños y niñas de sectores } \\
\text { de pobreza encuentren sus propias maneras de resolverlos. }\end{array}$ \\
\hline
\end{tabular}


doi: http://dx.doi.org/10.15359/ree.21-1.17

URL: http://www.una.ac.cr/educare

CORREO: educare@una.cr

Preguntas abiertas: estas fueron

1. ¿Indique 4 características del personal docente que enseña alfabetización inicial en contextos de pobreza?

2. Señala las 4 características de una escuela vulnerable.

\section{Procedimiento}

Antes de aplicar el instrumento se obtuvo la autorización firmada por parte de los sujetos responsables institucionales y luego se firmaron los consentimientos informados de cada participante. Para responder al cuestionario los sujetos ocuparon, en promedio, entre 20 y 35 minutos.

\section{Análisis}

En el análisis cualitativo, las respuestas a las preguntas abiertas fueron categorizadas por dos investigadoras en forma independiente. Luego se revisaron en conjunto y se compararon las categorías. A continuación, se ordenaron las categorías desde las más simples a las más complejas y se clasificaron como grupos de atributos que configuraron tres tipos de visiones: estereotipada, intermedia y compleja. Se categorizó individualmente y luego se calculó el índice Kappa, se encontró una buena concordancia interjueces (con valores entre 0,6 a 0,8), para cada uno de los grupos.

En el análisis cualitativo de las escalas Likert, se realizó un estudio factorial exploratorio con el método de extracción de componentes principales con rotación promax, por considerar que las variables están correlacionadas y así evidenciar la estructura interna del instrumento (Cook y Beckman, 2006), además de calcular el porcentaje de la varianza explicada por las dimensiones que emergieron (Macía, 2010). Por otra parte, se obtuvo evidencia de consistencia interna del cuestionario a través del índice alfa de Cronbach por cada una de las dimensiones.

Posteriormente, se transformaron las respuestas a los ítems de la escala Likert a puntajes Z por escala y se distribuyeron de acuerdo con +1DS y -1Ds, luego se realizó un análisis de conglomerados de K medias (ver Tabla 1) y se compararon los grupos mediante ANOVA. Para todos los cálculos se usó el software SPSS 17.0.

\section{Resultados}

\section{Análisis cualitativo}

\section{Buen profesor o profesora de alfabetización}

El análisis cualitativo arrojó tres visiones de buen profesor o profesora que enseña alfabetización inicial: 
doi: http://dx.doi.org/10.15359/ree.21-1.17

URL: http://www.una.ac.cr/educare

CORREO: educare@una.cr

Visión estereotipada: considera que la personalidad es la característica más importante de un profesor o profesora, por sobre las profesionales.

Visión intermedia: combinación entre ideas estereotipadas y valoración de conocimiento profesional del profesor o profesora.

Visión compleja: maneja conocimiento de la disciplina y de la didáctica.

Como vemos en la Tabla 3, la visión con mayor porcentaje de respuesta fue la visión intermedia, en la que se representa a un buen profesor o profesora mencionando algunas características profesionales combinadas con varios rasgos afectivo-motivacionales. A esta visión adhiere un $48.6 \%$ de estudiantes, un $44.4 \%$ de personal formador de docentes y un $31.9 \%$ del profesorado en servicio.

Sin embargo, un $39.1 \%$ del profesorado en servicio, más un $27 \%$ de estudiantes de pedagogía y un $26 \%$ del personal formador muestran una visión estereotipada del buen profesorado, centrada, principalmente, en aspectos emocionales. La visión compleja, en la que priman las características profesionales por sobre las afectivas, aparece en un $29.6 \%$ del personal formador, un $29 \%$ del profesorado en servicio y en un $23.2 \%$ en el estudiantado de pedagogía.

Tabla 3: Número y porcentaje de sujetos según visión sobre buen profesor o profesora de alfabetización

\begin{tabular}{ccccccc}
\hline \multirow{2}{*}{ Visión de buen profesor/a /tipo de sujeto } & \multicolumn{2}{c}{ Estudiantes } & \multicolumn{2}{c}{ Profesorado } & \multicolumn{2}{c}{ Personal formador } \\
\cline { 2 - 6 } & $\mathrm{N}$ & $\%$ & $\mathrm{~N}$ & $\%$ & $\mathrm{~N}$ & $\%$ \\
\hline Visión estereotipada & 75 & 28.3 & & 39.1 & 26.0 \\
Visión intermedia & 129 & 48.7 & & 31.9 & 44.4 \\
Visión compleja & 61 & 23.0 & & 29.0 & 29.6 \\
\hline
\end{tabular}

\section{Escuela vulnerable}

Al igual que respecto al buen profesor o profesora, se encontraron tres visiones respecto a la escuela vulnerable:

Visión estereotipada: destaca la carencia material y de infraestructura y una valoración negativa del estudiantado y sus familias.

Visión intermedia: combina algunas de las ideas estereotipadas más alguna consideración del profesorado o gestión de la escuela.

Visión compleja: tensiona factores causales más complejos tales como las políticas públicas, la sociedad reproductora o un problema del sistema. 
doi: http://dx.doi.org/10.15359/ree.21-1.17

URL: http://www.una.ac.cr/educare

Según vemos en la Tabla 4, al igual que en la pregunta anterior, predomina la visión intermedia en la que se señalan características negativas en familias, el estudiantado y escuela, combinadas con el reconocimiento del bajo compromiso, preparación y expectativas por parte del profesorado o personal directivo. En esta visión encontramos al $61.9 \%$ de estudiantes y al $52 \%$ de personal formador.

En cambio, el 69.7\% del profesorado en servicio presenta una visión estereotipada de la escuela vulnerable, destacando cualidades negativas tanto de la infraestructura como de las familias y alumnado. Llama la atención que un 36\% del personal formador también sostenga esta visión. Además, se observa muy baja frecuencia de la visión compleja caracterizada por un análisis crítico de las políticas públicas y del macrosistema. Esta baja frecuencia se hace evidente en que solo un $4.5 \%$ del profesorado y un $8.3 \%$ de estudiantes de pedagogía aluden a estos factores. Nuevamente sorprende que solo un $12 \%$ de los formadores y formadoras de docentes los mencione.

Tabla 4: Número y porcentaje de sujetos según visión sobre escuela vulnerable

\begin{tabular}{crrrrrr}
\hline \multirow{2}{*}{ Visión escuela vulnerable/tipo de sujeto } & \multicolumn{2}{c}{ Estudiante } & \multicolumn{2}{c}{ Profesorado } & \multicolumn{2}{c}{ Personal formador } \\
\cline { 2 - 7 } & \multicolumn{1}{c}{$\mathrm{N}$} & $\%$ & $\mathrm{~N}$ & $\%$ & $\mathrm{~N}$ & $\%$ \\
\hline Visión estereotipada & 79 & 29.8 & 46 & 69.7 & 9 & 36.0 \\
Visión intermedia & 164 & 61.9 & 17 & 25.8 & 13 & 52.0 \\
Visión compleja & 22 & 8.3 & 3 & 4.5 & 3 & 12.0
\end{tabular}

\section{Análisis cuantitativo}

\section{Creencias sobre alfabetización inicial}

Se condujo un análisis factorial exploratorio usando el método de extracción de componentes principales con rotación promax, según se observa en la Tabla 5.

Tabla 5: KMO y prueba de Bartlett Cuestionario de alfabetización inicial

\begin{tabular}{cccc}
\hline Kaiser-Meyer-Olkins & \multicolumn{3}{c}{ Prueba de esfericidad de Bartlett } \\
\hline \multirow{2}{*}{, 853} & Chi cuadrado aprox. & Grados de libertad & Significancia \\
\cline { 2 - 4 } & 3009,155 & 595 &, 000 \\
\hline
\end{tabular}


doi: http://dx.doi.org/10.15359/ree.21-1.17

URL: http://www.una.ac.cr/educare

CORREO: educare@una.cr

La solución factorial arrojó una estructura de cinco factores, correspondientes al 49,9\% de la varianza. Las escalas presentaron, en su mayoría, adecuados niveles de confiabilidad (ver Tabla 6).

Tabla 6: Componentes del Cuestionario de creencias sobre alfabetización inicial

\begin{tabular}{llc}
\hline \multicolumn{1}{c}{ Factor } & \multicolumn{1}{c}{ Items } & Alfa de Cronbach \\
\hline Visión tradicional de la alfabetización & $17,14,22,16,18,31$ &, 800 \\
Visión actual de la alfabetización & $28,27,25,29,24$ &, 713 \\
Prejuicio sobre alfabetización en contextos de pobreza & $15,4,20,11$ &, 673 \\
$\begin{array}{l}\text { Condiciones para la alfabetización en contextos de pobreza } \\
\text { Explicaciones del éxito o fracaso en la alfabetización en }\end{array}$ & $23,5,33,1$ &, 541 \\
contextos de pobreza & $9,35,7$ &, 514 \\
\hline
\end{tabular}

A continuación, se analizaron las creencias sobre alfabetización inicial utilizando las respuestas a estos 22 ítems, calculando los puntajes $Z$ de cada sujeto por escala y luego se distribuyeron entre -1DS y +1DS y luego se organizaron en 4 conglomerados de $\mathrm{K}$ medias (ver Tabla 7) y se compararon mediante ANOVA.

Tabla 7: Resumen de conglomerados de K medias en la escala de creencias sobre alfabetización inicial

\begin{tabular}{lcccc}
\hline & \multicolumn{4}{c}{ Conglomerados } \\
\cline { 2 - 5 } Escala & 1 & 2 & 3 \\
Intermedio & Complejo & Estereotipado & $\begin{array}{c}4 \\
\text { Intermedio }\end{array}$ \\
\hline Explicación éxito-fracaso &, 06149 &,- 15326 & 1,17586 &,- 67934 \\
Visión tradicional &, 55931 &,- 83522 &, 84459 &,- 91460 \\
Prejuicio sobre pobreza &,- 14980 &,- 29584 & 1,50289 &,- 52971 \\
Visión actualizada &, 33704 & $-1,81099$ &, 12564 &, 12506 \\
Condiciones alfabetización &, 50310 & $-1,31390$ &, 54091 &,- 50188 \\
\hline
\end{tabular}

El análisis estadístico muestra diferencias significativas entre conglomerados en las escalas de visión tradicional $(\mathrm{F}(2,353)=7.335, \mathrm{p}<.001)$ y visión actualizada $(\mathrm{F}(2,353)=5.213, \mathrm{p}<.006)$. No hubo diferencias significativas en las escalas prejuicio sobre la pobreza, condiciones para la alfabetización y explicaciones de éxito y fracaso en la alfabetización. 
doi: http://dx.doi.org/10.15359/ree.21-1.17

URL: http://www.una.ac.cr/educare

CORREO: educare@una.cr

Como puede observarse en la Tabla 8, la mayoría de participantes se concentra en una visión intermedia, es decir, el 72\% del estudiantado de pedagogía (conglomerado $1=41 \%$ y $4=31 \%$ ), el $71 \%$ del profesorado en servicio (conglomerado $1=45 \%$ y $4=26 \%$ ) y el $76 \%$ del personal formador (conglomerado 4=48\% y 1=28\%). En esta visión intermedia el conglomerado 1 se encuentra más orientado al polo tradicional y el conglomerado 4 se inclina más hacia el polo complejo.

El conglomerado 3 es el más tradicional, con creencias estereotipadas frente a la escala de explicación del éxito-fracaso (lo que quiere decir que estos sujetos valoran el papel de la evaluación homogénea y de la estimulación de la velocidad lectora) y la escala prejuicio sobre la pobreza (es decir, que los sujetos creen en el efecto negativo de la diversidad de estudiantes, de sus habilidades lingüísticas y de la baja escolaridad de los padres y madres). En este grupo se encuentra el $26 \%$ del profesorado en servicio, el $15 \%$ del estudiantado y el $12 \%$ de formadores y formadoras de profesores.

Tabla 8: Distribución de los grupos por conglomerado en creencias sobre alfabetización inicial

\begin{tabular}{lcccc}
\hline \multicolumn{1}{c}{ Sujetos } & \multicolumn{4}{c}{ Conglomerados } \\
\cline { 2 - 5 } & $\begin{array}{c}\text { Intermedio } \\
\mathrm{f}(\%)\end{array}$ & $\begin{array}{c}\text { Complejo } \\
\mathrm{f}(\%)\end{array}$ & $\begin{array}{c}\text { Estereotipado } \\
\mathrm{f}(\%)\end{array}$ & $\begin{array}{c}\text { Intermedio } \\
\mathrm{f}(\%)\end{array}$ \\
\hline Estudiantes de pedagogía & $109(41 \%)$ & $34(13 \%)$ & $41(15 \%)$ & $81(31 \%)$ \\
Profesorado en servicio & $30(45 \%)$ & $2(3 \%)$ & $17(26 \%)$ & $17(26 \%)$ \\
Personal formador de profesorado & $7(28 \%)$ & $3(12 \%)$ & $3(12 \%)$ & $12(48 \%)$ \\
Totales & 146 & 41 & 61 & 110 \\
\hline
\end{tabular}

Según se observa en la Tabla 8, el conglomerado 2 representa la visión más compleja y en este resaltan, en primer lugar, las diferencias en la escala denominada visión compleja. Esta implica creer que la alfabetización, la disposición de bibliotecas y la superación de las actividades de comprensión literal amplían las posibilidades de desarrollo cognitivo y social de los niños y las niñas en contextos de pobreza. En segundo lugar, en este mismo conglomerado se observa una visión crítica de las afirmaciones que aparecen en la escala condiciones para la alfabetización, la que se refiere a ciertos preceptos sobre lo que es importante a la hora de alfabetizar: existencia de libros en las casas, refuerzo de la familia en casa, procurar formar grupos homogéneos y presentar textos en español estándar. A pesar de ser el conglomerado más complejo, en este solo se concentra un $13 \%$ de estudiantes, un $12 \%$ de formadores y formadoras de profesores y un 3\% del profesorado en servicio. 
doi: http://dx.doi.org/10.15359/ree.21-1.17

URL: http://www.una.ac.cr/educare

CORREO: educare@una.cr

\section{Creencias sobre el aprendizaje de la matemática inicial}

Con los datos obtenidos se condujo un análisis factorial exploratorio usando el método de extracción de componentes principales con rotación promax que se observa en la Tabla 9, por asumir que los datos están correlacionados entre sí, para así evidenciar estructura interna del instrumento.

Tabla 9: KMO y prueba de Bartlett cuestionario de matemática

\begin{tabular}{|c|c|c|c|}
\hline \multirow[t]{2}{*}{ Kaiser-Meyer-Olkins } & \multicolumn{3}{|c|}{ Prueba de esfericidad de Bartlett } \\
\hline & Chi cuadrado aprox. & Grados de libertad & Significancia \\
\hline ,862 & 3828,809 & 595 & ,000 \\
\hline
\end{tabular}

De esta manera, el análisis factorial dio una solución de seis factores, correspondientes al $56,43 \%$ de la varianza ${ }^{5}$. Como se observa en la Tabla 10, en general las escalas presentaron adecuados niveles de confiabilidad.

Tabla 10: Composición del Cuestionario de creencias sobre la enseñanza y aprendizaje de la matemática

\begin{tabular}{llc}
\hline \multicolumn{1}{c}{ Factor } & \multicolumn{1}{c}{ Ítems } & Alfa de Cronbach \\
\hline Prejuicio sobre matemática y pobreza & $8,28,3,23,35,7,25,22$ &, 800 \\
Representación de la pobreza & $17,11,26,24,5,14$ &, 814 \\
Formación del profesorado & 15,21 &, 767 \\
Factores de éxito & $32,33,34$ &, 535 \\
Determinismo de la pobreza & $1,2,9$ &, 631 \\
Aprendizaje de las matemáticas & 29,30 &, 635 \\
\hline
\end{tabular}

Las creencias acerca de la enseñanza y el aprendizaje de la matemática inicial se evaluaron con un cuestionario con 24 ítems. Se organizaron 4 conglomerados de K medias (ver Tabla 11) y se compararon mediante ANOVA.

${ }^{5}$ Este es un valor aproximado, ya que al usarse una rotación que supone correlación entre los factores, las sumas de los cuadrados de las saturaciones no se pueden añadir para obtener una varianza total. 
doi: http://dx.doi.org/10.15359/ree.21-1.17

URL: http://www.una.ac.cr/educare

CORREO: educare@una.cr

Tabla 11: Síntesis de los conglomerados de K medias en la escala de creencias sobre matemática

\begin{tabular}{|c|c|c|c|c|}
\hline \multirow[b]{2}{*}{ Escala } & \multicolumn{4}{|c|}{ Conglomerados } \\
\hline & $\begin{array}{c}1 \\
\text { Estereotipado }\end{array}$ & $\begin{array}{c}2 \\
\text { Intermedio }\end{array}$ & $\begin{array}{c}3 \\
\text { Complejo }\end{array}$ & $\begin{array}{c}4 \\
\text { Intermedio }\end{array}$ \\
\hline Prejuicio matemática/pobreza & 1,0427 & 0441 &,- 9180 &,- 4360 \\
\hline Representación pobreza & 1,0786 & 1604 & $-1,0449$ &,- 6917 \\
\hline Formación del profesorado & 0799 &,- 3056 & 1840 & 4829 \\
\hline Factores de éxito & 8221 & 1648 &,- 8196 &,- 5972 \\
\hline Determinismo pobreza & 8872 & 1918 &,- 8118 &,- 7062 \\
\hline Aprendizaje matemática &,- 0419 & 1034 & $-1,0744$ & 7100 \\
\hline
\end{tabular}

Como se observa en la Tabla 11, el conglomerado 1 es el más estereotipado y el conglomerado 3 el más complejo. El análisis cuantitativo muestra diferencias significativas entre los cuatro conglomerados en todas las escalas, es decir, en la escala prejuicio pobreza/matemática $(F(2,352)=$ $110.538, p<.000)$, en representación de la pobreza $(F(2,352)=159.678, p<.000)$, en formación del profesorado $(\mathrm{F}(2,352)=14.233, \mathrm{p}<.000)$, en factores de éxito $(\mathrm{F}(2,352)=68.775, \mathrm{p}<.000)$, en determinismo de la pobreza $(F(2,352)=90.053, p<.000)$ y en aprendizaje de la matemática $(F(2,352)=56.542, p<.000)$.

En la Tabla 12 vemos que destaca el conglomerado 2 con la mayor concentración de sujetos (142), en el que se observa una visión claramente promedio, principalmente entre el profesorado en servicio (43\%) pero también de estudiantes de pedagogía y formadores y formadoras de profesores (39\%). Si a este resultado le sumamos el conglomerado 4, encontramos que la mayor parte de los sujetos, es decir sobre el $50 \%$ de cada grupo participante, se encuentra en esta visión.

Tabla 12: Distribución de los grupos por conglomerado en creencias sobre matemática

\begin{tabular}{lcccc}
\hline & \multicolumn{4}{c}{ Conglomerados } \\
\cline { 2 - 5 } \multicolumn{1}{c}{ Sujetos } & $\begin{array}{c}1 \\
\text { Estereotipado } \\
\mathrm{f}(\%)\end{array}$ & $\begin{array}{c}2 \\
\text { Intermedio } \\
\mathrm{f}(\%)\end{array}$ & $\begin{array}{c}3 \\
\text { Complejo } \\
\mathrm{f}(\%)\end{array}$ & $\begin{array}{c}\text { Intermedio } \\
\mathrm{f}(\%)\end{array}$ \\
\hline Estudiantes de pedagogía & $56(21 \%)$ & $104(39 \%)$ & $47(18 \%)$ & $58(22 \%)$ \\
Profesores en servicio & $22(35 \%)$ & $27(43 \%)$ & $6(9 \%)$ & $8(13 \%)$ \\
Formadores de profesores & $5(18 \%)$ & $11(39 \%)$ & $5(18 \%)$ & $7(19 \%)$ \\
Totales & 83 & 142 & 58 & 73 \\
\hline
\end{tabular}


doi: http://dx.doi.org/10.15359/ree.21-1.17

URL: http://www.una.ac.cr/educare

CORREO: educare@una.cr

La visión compleja es representada por el conglomerado $3 \mathrm{y}$, al igual que en el caso de las creencias sobre alfabetización, corresponde al grupo más pequeño (58 sujetos) en el que solo participa el $19 \%$ de formadores y formadoras de profesores y el $18 \%$ de estudiantes y un $9 \%$ del profesorado. En este grupo sobresalen las creencias más actualizadas respecto a la representación de la pobreza, superando los prejuicios sobre las familias y la niñez, y del aprendizaje de la matemática, creyendo en la estimulación de distintas formas de resolver problemas y en superar la visión negativa de las familias.

Al contrario, el conglomerado 1 representa al grupo más tradicional, en el que predominan los profesores en servicio (35\%), pero también se observa un $21 \%$ de estudiantes y un $18 \%$ de formadores y formadoras de profesores. Aquí se observa una visión estereotipada en escala prejuicio sobre la pobreza (creencia en la inutilidad de la matemática para los niños de contextos de pobreza y en la escala representaciones de los pobres, en la que se destacan aspectos negativos de las familias y la niñez provenientes de sectores de pobreza, tales como: deterioro familiar, problemas conductuales y emocionales, escasa valoración, importancia y apoyo de la familia respecto al aprendizaje de la matemática.

\section{Discusión y conclusiones}

Con respecto a nuestra pregunta de estudio, podemos concluir que existen diferencias en las creencias que posee el estudiantado de pedagogía, el profesorado en servicio y también los formadores y formadoras de docentes, pero no se observa predominio de un tipo de creencias en un grupo particular, es decir, los tres grupos se distribuyeron en los distintos tipos de visiones, desde las más simples a las más complejas, aunque esta última siempre presentó los porcentajes más bajos. Este hallazgo tiene importantes implicancias, pues demuestra que se requiere realizar acciones para modificar las creencias en los tres tipos de participantes.

Respecto a las creencias sobre la escuela vulnerable, el análisis cualitativo muestra que, a diferencia del estudiantado de pedagogía y los formadores y formadoras de docentes -quienes sostienen una visión intermedia respecto de las escuelas en contextos de pobreza- el profesorado en servicio posee mayoritariamente una visión estereotipada. Este último grupo, rara vez alude en sus explicaciones a sí mismo -a diferencia del estudiantado y los formadores y formadoras- $y$ cuando lo hacen, destacan el esfuerzo y sacrificio que realizan por sus estudiantes. Al contrario, tienden resaltar principalmente la falta de infraestructura adecuada, la escasez de recursos, el tipo de alumnado problemático que reciben y la falta de apoyo de las familias. Estos resultados han sido reportados también en estudios anteriores realizados por Román (2003) y Cuadra (2009). De modo que aquí nos encontramos frente a una barrera importante porque el profesorado no ve posibilidades de cambio, considerando que son estas condiciones las que explican el inevitable fracaso de la niñez más vulnerable. Para resolver esta situación, Román (2003) sugiere que debemos 
trabajar "en identificar y proporcionar los recursos y condiciones que permitan la "innovación" de la Representación Social [del profesor] [sobre el] alumno pobre y excluido" (p. 127).

Por otra parte, se observa una falta de análisis crítico en todos los grupos, ya que el porcentaje que alude a problemas en las políticas públicas o del sistema social más amplio, incluso entre los formadores y formadoras de profesorado, es bastante bajo. Sin duda, estas visiones podrían estar estimulando bajas expectativas respecto a las posibilidades de éxito escolar de la niñez en contextos de pobreza en todos los ámbitos de la formación docente.

Respecto a la preparación que debiera tener un buen profesor o profesora, también observamos que entre los participantes predomina la visión intermedia, en la que la mayoría resalta los rasgos de personalidad, aunque también reconoce la necesidad de algún tipo de conocimiento profesional. La visión compleja, en cambio, solo se presenta en alrededor de un tercio de la muestra.

El análisis de conglomerados de los cuestionarios nos informa que, respecto a la alfabetización, predomina la visión intermedia en los sujetos, en la que se combinan creencias tradicionales y actualizadas en las escalas de visión tradicional, visión actualizada, prejuicio sobre la pobreza, condiciones para la alfabetización y explicaciones de éxito y fracaso en la alfabetización inicial. Lo mismo ocurre en el caso de las creencias sobre enseñanza y aprendizaje de la matemática inicial. En esta categoría intermedia también se combinan creencias más tradicionales con las más complejas en las escalas de prejuicio sobre pobreza/matemática, representación de la pobreza, formación del profesorado, factores de éxito, determinismo de la pobreza y aprendizaje de la matemática. En ambas escalas, la visión estereotipada es levemente mayor a la visión compleja, siendo esta última la menos presente entre los sujetos.

Respecto al objetivo de caracterizar las creencias de las distintas personas actoras, nuestros resultados nos muestran que, respecto a las creencias del profesorado en servicio, se observa el predominio de la visión intermedia en las creencias de alfabetización y matemática inicial, aunque más inclinado hacia el polo simple. No obstante, en el análisis cualitativo nos muestra un predominio de la visión estereotipada, por ejemplo, respecto al buen profesor o profesora presentaron mayor tendencia a valorar los aspectos de personalidad por sobre los profesionales y respecto a la escuela vulnerable casi el 70\% definió la escuela vulnerable a través de estereotipos de la niñez y sus familias. Según nuestros datos, el profesorado fue el grupo que presentó menor porcentaje de creencias complejas.

Respecto a estudiantes que se encuentran cursando actualmente carreras de pedagogía, se observan creencias bastante negativas a pesar de encontrarse en pleno proceso de formación pedagógica. Por ejemplo, respecto a la escuela vulnerable sobre el $60 \%$ posee una visión intermedia, combinando principalmente factores relacionados con infraestructura y recursos, características de la niñez y sus familias con algunos rasgos pedagógicos de las escuelas. Denegri et al. (2010), en una muestra universitaria de 121 estudiantes de cuarto año de pedagogía y medicina, encontraron que 
doi: http://dx.doi.org/10.15359/ree.21-1.17

URL: http://www.una.ac.cr/educare

CORREO: educare@una.cr

estos mostraron dificultad para diferenciar entre pobre y pobreza, tendiendo a construir significados orientados a lo concreto y lo personificado. Los autores señalan preocupación por estas ideas en estudiantes que debieran tener especial contacto con estas poblaciones.

Por otra parte, el grupo de estudiantes de nuestro estudio posee creencias intermedias respecto a alfabetización y matemática inicial, aunque en ambos casos se inclina más hacia el polo simple de las creencias que hacia el complejo. Otros estudios encuentran resultados que complementan los nuestros, por ejemplo, el informe TEDS-M (Tatto, et al., 2012), que exploró el nivel de preparación de los futuros profesores y profesoras para enseñar matemáticas en 17 países -entre los que se encontraba Chile con uno de los más bajos desempeños del grupo total de participantes- señala que hubo una tendencia general a que estudiantes de pedagogía con menor conocimiento de la matemática creyeran que esta asignatura es un conjunto de reglas y procedimientos, que su aprendizaje requiere seguir las instrucciones del profesorado y que la matemática es una habilidad fija. Además, plantea que, casi sin excepción, las creencias de los futuros profesores y profesoras en cada país coincidían con el patrón de creencias de sus formadores y formadoras, lo que sugiere que el cambio sería poco probable a menos que ocurra primero un cambio en las creencias que sostienen los formadores y formadoras de docentes.

Finalmente, respecto a estos formadores y formadoras, se esperaba que fuera el grupo que presentara creencias con predominio de creencias más complejas. Nuestros resultados muestran que esto no fue así, muy por el contrario, fue sorprendentemente bajo. Esto quiere decir que los formadores y formadoras de docentes todavía presenta una comprensión insuficiente para estar en condiciones de transformar la visión estereotipada de sus estudiantes sobre la pobreza y romper el círculo vicioso del fracaso escolar en estos contextos.

Este hecho tiene importantes implicancias y pone de relieve la necesidad de formar a los formadores y formadoras de profesores. Según Lunenberg (2002), este es un aspecto del ecosistema que parece haber sido omitido de la investigación. Murray y Male (2005) muestran que la mayoría de los formadores y formadoras ha aprendido a través de la práctica no estructurada, en solitario y dependiendo de su esfuerzo individual. Sin embargo, necesitan ayuda para 'cambiar los lentes' que poseen para resolver las demandas de la formación del profesorado; necesitan conocer explícitamente y mostrar apertura al análisis de los supuestos, creencias y prácticas personales; también necesitan tiempo, espacio y oportunidades para reflexionar y analizar sus prácticas y las preguntas, temas y dilemas que estas generan; además, necesitan oportunidades para discutir con sus pares, con la dirección de personas mentoras expertas y experimentadas con quienes trabajar de cerca (Cochran-Smith, 2003; Murray y Male, 2005).

Según Gorski (2012), cuando no conocemos, usamos estereotipos para rellenar los espacios en blanco. El peligro de no atender a esta demanda podría profundizar los efectos negativos, ya que como señala Burrell (2012), "lack of experience with diversity can cause misunderstandings 
among social groups, especially if the information learned about one another comes from unreliable sources that perpetuate stereotypes, prejudice, and bias [la falta de experiencia con la diversidad puede causar falta de comprensión entre grupos sociales, especialmente si la información aprendida sobre los otros viene de fuentes no confiables que perpetúan los estereotipos, el prejuicio y el sesgo]" (p. 547).

Un programa de formación de profesorado debiera implementar varias estrategias para preparar mejor estudiantes de pedagogía para satisfacer las necesidades de la niñez en contextos de pobreza. Por ejemplo, debieran examinar las metas y la misión de sus programas; evaluar la relevancia y factibilidad del currículo para lograr sus metas; $y$, finalmente, evaluar a los equipos formadores de docentes para determinar si estos poseen las habilidades, el conocimiento, y compromiso requeridos para lograr las metas propuestas (Hughes, 2010). En un estudio reciente Cho et al. (2015) desarrollaron una estrategia interdisciplinaria para agregar al currículo dos módulos on-line para cultivar en estudiantes de pedagogía una comprensión de la pobreza y desarrollar en ellos y ellas las competencias necesarias para entregar ambientes de aprendizaje sensibles a las necesidades de la niñez proveniente de contextos de pobreza. Los módulos se centraron en 1) el profesor o profesora que facilita el aprendizaje y 2) el profesor o profesora como antropólogo. Sus resultados muestran que, aunque el estudiantado valora la introducción de estos módulos, gran parte continuaba con una visión de la pobreza centrada en el déficit. Los autores y autoras recomiendan el uso de estas estrategias, además de procurar que 1) se dé más tiempo a cada módulo, 2) se usen en las etapas iniciales y avanzadas de la formación y, 3) se ofrezcan múltiples oportunidades de tener experiencias académicas para comprender la pobreza.

Usando las palabras de Gorski, (2012, p. 314):

How are we, as educators, socialized to contribute to class inequities even when our intention is to counter them?... we cannot hope to provide the best possible educational experiences to students from families in poverty without a willingness to reject sterereotypes and prejudices. [¿Cómo estamos nosotros, como educadores, socializados para contribuir a las desigualdades de clase, incluso cuando nuestra intención es hacerle frente?... no podemos esperar entregar las mejores experiencias educativas a los estudiantes de familias en situación de pobreza, sin la voluntad de rechazar los estereotipos y prejuicios que poseemos].

Por otra parte, existe una limitada experticia en los formadores y las formadoras (Gorski, 2009). Los programas de formación del profesorado debieran hacer mayores esfuerzos para reflexionar sobre este tema de modo que los equipos a cargo de la formación de docentes avancen en sus creencias y alcancen una conceptualización y práctica más adecuadas acerca de los desafíos, oportunidades y estrategias para trabajar en contextos de pobreza. 
doi: http://dx.doi.org/10.15359/ree.21-1.17

URL: http://www.una.ac.cr/educare

CORREO: educare@una.cr

\section{Referencias}

Agencia de Calidad de la Educación. (2015). Entrega de resultados de aprendizaje 2014. Simce e indicadores dedesarrollopersonalysocial. Recuperado de http://archivos.agenciaeducacion. cl/resultados-2014/Presentacion Entrega Resultados 2014.pdf

Añorve, G. (2011) Las teorías implícitas de los docentes normalistas sobre la alfabetización inicial. Ponencia presentada en el I/ Congreso Internacional de Investigación Educativa: Su incidencia en la realidad social. San José, Costa Rica: Universidad de Costa Rica, INIE. Recuperado de http://www.comie.org.mx/congreso/memoriaelectronica/v11/docs/ area 15/0502.pdf

Armstrong, A. (2010). Myths of poverty. Realities for students. The Educational Digest, 75(8), 49-53.

Belleï, C. (2001). El talón de Aquiles de la reforma. Análisis sociológico de la política de los 90 hacia los docentes en Chile. En S. Martinic y M. Pardo (Eds.), Economía política de las reformas educativas en América Latina (pp. 129-146). Santiago, Chile: PREAL-CIDE. Recuperado de http://cippec.org/mapeal/wp-content/uploads/2014/06/MARTINIC-yPARDO-Econom\%C3\%ADa-pol\%C3\%ADtica-de-las-reformas-educativas-en-AmLat.pdf

Beswick, K. (2012). Teachers'beliefs about school mathematics and mathematicians'mathematics and their relationship to practice. Educational Studies in Mathematics, 79, 127-147. doi: https://doi.org/10.1007/s10649-011-9333-2

Bryan, L. A. y Atwater, M. M. (2002). Teacher beliefs and cultural models: A challenge for science teacher preparation programs. Science Teacher Education, 86(6), 821-839. doi: https://doi. org/10.1002/sce. 10043

Burrell, S. (2012). Preparing students for social action in a social justice education course: What works? Equity \& Excellence in Education, 45(4), 547-560. doi: https://doi.org/10.1080/10665 $\underline{684.2012 .719424}$

Carlson, B. A. (2000). ¿Qué nos enseñan las escuelas sobre la educación de los niños pobres en Chile? Revista de la CEPAL, 72, 165-184. Recuperado de http://repositorio.cepal.org/ bitstream/handle/11362/10713/1/072165184 es.pdf

Cho, M.-H., Convertino, C. y Khourey-Bowers, C. (2015, Springer). Helping preservice teachers (PSTs) understand the realities of poverty: Innovative curriculum modules. Educational Technology Research \& Development, 63(2), 303-324. doi: https://doi.org/10.1007/s11423$\underline{015-9366-9}$

Cochran-Smith, M. (2003). Learning and unlearning:The education of teacher educators. Teaching and Teacher Education, 19(1), 5-28. doi: https://doi.org/10.1016/S0742-051X(02)00091-4 
doi: http://dx.doi.org/10.15359/ree.21-1.17

URL: http://www.una.ac.cr/educare

CORREO: educare@una.cr

Condemarín, M. (2002). Falsas concepciones sobre la competencia lingüística, los valores y la cultura de los niños y niñas provenientes de familias pobres. Lectura y Vida, 23(1), 1622. Recuperado de http://www.lecturayvida.fahce.unlp.edu.ar/numeros/a23n1/23 01 Condemarin.pdf

Cook, D. A. y Beckman, T. J. (2006). Current concepts in validity and reliability for psychometric instruments: Theory and application. The American Journal of Medicine, 119(2), 166.e167166.e16. doi: https://doi.org/10.1016/j.amjmed.2005.10.036

Cuadra, D. (2009). Teorías subjetivas en docentes de una escuela de bajo rendimiento, sobre la enseñanza y el aprendizaje del alumno. Revista Mexicana de Investigación Educativa, 14(42), 939-967. Recuperado de http://www.scielo.org.mx/scielo.php?script=sci arttext\& pid=S1405-66662009000300015

Del Río, F. y Balladares, J. (2010). Género y nivel socioeconómico de los niños: Expectativas del docente en formación. Psykhe, 19(2), 81-90. doi: https://doi.org/10.4067/S0718$\underline{22282010000200008}$

Denegri, M., Cabezas, D., Sepúlveda, J., Del Valle, C., González, Y. y Miranda, H. (2010). Representaciones sociales sobre pobreza en estudiantes universitarios chilenos. Liberabit. Revista de Psicología, 16(2), 161-170. Recuperado de http://www.redalyc.org/articulo. oa?id=68617161005

Elkind, D. (2004). The problem with constructivsm. The Educational Forum, 68(4), 306-312. doi: https://doi.org/10.1080/00131720408984646

Eyzaguirre, B. (2004, verano). Claves para la educación en pobreza. Estudios Públicos, 93, 249-277. Recuperado de http://www.cepchile.cl/cep/site/artic/20160304/ asocfile/20160304093204/r93eyzaguirre claves para.pdf

Gil, F. y Rico, L. (2003). Concepciones y creencias del profesorado de secundaria sobre enseñanza y aprendizaje de las matemáticas. Enseñanza de las Ciencias, 21(1), 27-47. Recuperado de http://ddd.uab.cat/pub/edlc/02124521v21n1/02124521v21n1p27.pdf

Gorski, P. (2009). What we're teaching teachers: An analysis of multicultual teacher education coursework syllabi. Teaching and Teacher Education, 25(2), 309-318. doi: https://doi. org/10.1016/j.tate.2008.07.008

Gorski, P. (2012). Perceiving the problem of poverty and schooling: Deconstructing the class stereotypes that mis-shape education practice and policy. Equity \& Excellence in Education, 45(2), 302-319. doi: https://doi.org/10.1080/10665684.2012.666934 
doi: http://dx.doi.org/10.15359/ree.21-1.17

URL: http://www.una.ac.cr/educare

CORREO: educare@una.cr

Hughes, J. A. (2010). What teacher preparation programs can do to better prepare teachers to meet the challenges of educating students living in poverty. Action in Teacher Education, 32(1), 54-64. doi: https://doi.org/10.1080/01626620.2010.10463542

Jadue, G. (1997). Factores ambientales que afectan el rendimiento escolar de los niños provenientes de familias de bajo nivel socioeconómico y cultural. Estudios pedagógicos, 23, 75-80. doi: https://doi.org/10.4067/S0718-07051997000100007

Jones, S. y Vagle, M. (2013). Living contradictions and working for change: Toward a theory of social class-sensitive pedagogy. Educational Researcher, 42(3), 129-141. doi: https://doi. org/10.3102/0013189X13481381

Joram, E. y Gabriele, A. J. (1998). Preservice teachers' prior beliefs: Transforming obstacles into opportunities. Teaching and TeacherEducation, 14(2), 175-191.doi: https://doi.org/10.1016/ S0742-051X(97)00035-8

Kagan, D. M. (1992). Implications of research on teacher belief. Educational Psychologist, 27(1), 65-90. doi: https://doi.org/10.1207/s15326985ep2701 6

Kuntze, S. (2012). Pedagogical content beliefs: Global, content domain-related and situationspecific components. Educational Studies in Mathematics, 79(2), 273-292. doi: https://doi. org/10.1007/s10649-011-9347-9

Levin, B. (1995). Educational responses to poverty. Canadian Journal of Education, 20(2), 211 224. Recuperado de https://doi.org/10.2307/1495278

Leyens, J.-P., Yzerbyt, V. y Schadron, G. (1994). Stereotypes and social cognition. London: Sage Publications.

Leyva, L. (2005) El currículum como espacio de legitimización de la cultura del pobre. Cuadernos Interculturales, 3(4). 27-36. Recuperado de http://www.redalyc.org/articulo. oa?id=55200403

Lortie, D. C. (1975). Schoolteacher: A sociological study. Chicago: University of Chicago Press.

Lunenberg, M. (2002). Designing a curriculum for teacher educators. European Journal of Teacher Education, 25(2-3), 263-277. doi: https://doi.org/10.1080/0261976022000044872

Macía, F. (2010). Validez de los tests y el análisis factorial: Nociones generales. Ciencia \& Trabajo, 12(35), 276-280. Recuperado de http://documents.mx/documents/validez-de-los-tests-yel-analisis-factorial-nociones-generales.html

Markovits, Z. (2011). Beliefs hold by pre-school prospective teachers toward mathematics and its teaching. Procedia Social and Behavioral Sciences, 11, 117-121. doi: https://doi. org/10.1016/j.sbspro.2011.01.045 
doi: http://dx.doi.org/10.15359/ree.21-1.17

URL: http://www.una.ac.cr/educare

CORREO: educare@una.cr

Ministerio de Desarrollo Social (2012). Informe de política social 2012. http://www. ministeriodesarrollosocial.gob.cl/ipos-2012/media/ipos 2012 pp 12-29.pdf

Murray, J. y Male, T. (2005). Becoming a teacher educator: Evidence from the field. Teaching and Teacher Education, 21(2), 125-142. doi: https://doi.org/10.1016/j.tate.2004.12.006

Nespor, J. (1987). The role of beliefs in the practice of teaching. Journal of curriculum studies, 19(4), 317-328. doi: https://doi.org/10.1080/0022027870190403

Pajares, M. F. (1992). Teachers' beliefs and educational research: Cleaning up a messy construct. Review of Educational Research, 62(3), 307-332. Doi: https://doi. org/10.3102/00346543062003307

Philipp, R. A., Ambrose, R., Lamb, L. L. C., Sowder, J. T., Schappelle, B. P., Sowder, L., ..., Chauvot, J. (2007). Effects of early field experiences on the mathematical content knowledge and beliefs of prospective elementary school teachers: An experimental study. Journal for Research in Mathematics Education, 38(5), 438-476. Recuperado de http://www.jstor.org/ stable/30034961

Raczynski, D. y Muñoz, G. (2005). Efectividad escolar y cambio educativo en condiciones de pobreza en Chile. Santiago, Chile: Ministerio de Educación. Recuperado de http://www.gse.upenn. edu/pdf/Efectividad\%20Escolar.pdf

Richardson, V. (2003). Preservice teachers' beliefs. En J. Raths y A. C. McAninch (Eds.), Teacher beliefs and classroom performance: Teacher impact of teacher education. Advances in Teacher Education (pp. 1-22). Greenwich, CT: Information Age Publishers.

Rodrigo, M. J., Rodríguez, A. y Marrero, J. (1993). Las teorías implícitas: Una aproximación al conocimiento cotidiano. Madrid: Visor.

Román, M. (2003). ¿Por qué los docentes no pueden desarrollar procesos de enseñanza aprendizaje de calidad en contextos sociales vulnerables? Persona y Sociedad, 17(1), 113-128. Recuperado de http://biblioteca.uahurtado.cl/ujah/pys/docs/2003/abril/17 1 pp113 128.pdf

Román, M. (2006). El desafío de aprender a leer, comprender y razonar en escuelas vulnerables. Pensamiento educativo, 39(2), 69-86. Recuperado de http://www.pensamientoeducativo. uc.cl/files/journals/2/articles/385/public/385-875-1-PB.pdf

Rosales, E. (2008). Concepciones y creencias docentes sobre el éxito y fracaso en el área curricular de comunicación integral (Tesis de licenciatura). Pontificia Universidad Católica del Perú. Lima. Recuperado de http://tesis.pucp.edu.pe/repositorio/bitstream/ handle/123456789/422/ROSALES CORDOVA ELIZABETH CONCEPCIONES CREENCIAS. pdf? sequence $=1$ \&isAllowed $=y$ 
doi: http://dx.doi.org/10.15359/ree.21-1.17

URL: http://www.una.ac.cr/educare

CORREO: educare@una.cr

Schadron, G. e Yzerbyt, V. (1996). Estereotipos y juicio social. En R. Y. Bourhis y J. P. Leyens, F. Morales y D. Páez (Eds.), Estereotipos, discriminación y relaciones entre grupos (pp. 113-137). Madrid: Mc Graw-Hill.

Shaw, D. M. y Mahlios, M. (2011). Literacy metaphors of pre-service teachers: Do they change after instruction? Which metaphors are stable? How do they connect to theories? Journal of Education for Teaching, 37(1), 77-92. doi: https://doi.org/10.1080/02607476.2011.538274

Stevens, D. D. y Palincsar, A. S. (1992). Urban teachers' beliefs and knowledge about literacy teaching and learning: An examination from mechanistic and contextualistic perspectives. Ponencia presentada en Annual Meeting of the American Educational Research Association. San Francisco. Recuperado de http://files.eric.ed.gov/fulltext/ED348452.pdf

Swars, S., Hart, L. C., Smith, S., Smith, M. E. y Tolar, T. (2007). A longitudinal study of elementary pre-service teachers' mathematics beliefs and content knowledge. School Science and Mathematics, 107(8), 325-335. doi: https://doi.org/10.1111/j.1949-8594.2007.tb17797.x

Szydlik, J., Szydlik, S. D. y Benson, S. R. (2003). Exploring changes in pre-service elementary teachers' mathematical beliefs. Journal of Mathematics Teacher Education, 6(3), 253-279. doi: https://doi.org/10.1023/A:1025155328511

Tatto, M. T., Peck, R., Schwille, J., Bankov, K., Senk, L., Rodriguez, M., ... Rowley, G. (2012). Policy, practice, and readiness to teach primary and secondary mathematics in 17 countries.Findings from the IEA teacher education and development study in mathematics (TEDS-MM). Amsterdam: International Association for the Evaluation of Educational Achievement. Recuperado de http://www.iea.nl/ fileadmin/user upload/Publications/Electronic versions/TEDS-M International Report.pdf

Templeton, S. (2011-2012). Teaching and learning morphology: A reflection on generative vocabulary instruction. The Journal of Education, 192(2-3), 101-107. Recuperado de http:// www.jstor.org/stable/42744238

Toluk-Uçar, Z., Pişkin, M., Akkaş, E. N. yTaşçi, D. (2010). İlköğretim Öğrencilerinin Matematik, Matematik Öğretmenleri ve Matematikçler Hakkındaki İnançları. Elementary students' beliefs about mathematics, mathematics' teachers and mathematicians. Eğitim Ve Bilim, 35(155), 131-144. Recuperado de http://egitimvebilim.ted.org.tr/index.php/EB/article/viewFile/568/54

Wideen, M., Mayer-Smith, J. y Moon, B. (1998). A critical analysis of the research on learning to teach: Making the case for an ecological perspective on inquiry. Review of Educational Research, 68(2), 130-131. doi: https://doi.org/10.3102/00346543068002130

Zakaria, E. y Musiran, N. (2010). Beliefs about the nature of mathematics, mathematics teaching and learning among trainee teachers. The Social Sciences, 5(4), 346-351. doi: https://doi. org/10.3923/sscience.2010.346.351 